\title{
Topological mirror symmetry for rank two character varieties of surface groups
}

\author{
Mirko Mauri ${ }^{1}$
}

Received: 15 January 2021 / Published online: 21 August 2021

(C) The Author(s) 2021

\section{Abstract}

The moduli spaces of flat $\mathrm{SL}_{2}$ - and $\mathrm{PGL}_{2}$-connections are known to be singular SYZ-mirror partners. We establish the equality of Hodge numbers of their intersection (stringy) cohomology. In rank two, this answers a question raised by Tamás Hausel in Remark 3.30 of "Global topology of the Hitchin system".

Keywords Intersection cohomology · Mirror symmetry $\cdot$ Character variety · E-polynomial $\cdot$ Perverse filtration

Mathematics Subject Classification $14 \mathrm{~J} 33 \cdot 14 \mathrm{D} 20$

Let $C$ be a compact Riemann surface of genus $g$ with base point $c \in C$, and $G$ be either $\mathrm{SL}_{r}$ or $\mathrm{PGL}_{r}$. We study the following moduli spaces (cf [14]):

- the de Rham moduli space of principal flat $G$-bundles on $C \backslash c$ with holonomy $e^{2 \pi i d / r}$ around $c$;

- the Dolbeault moduli space of semistable $G$-Higgs bundles of degree $d$, i.e. semistable pairs $(E, \phi)$ consisting of a principal $G$-bundle $E$ of degree $d$ and a section $\phi \in H^{0}\left(C, \operatorname{ad}(E) \otimes K_{C}\right)$, where $K_{C}$ is the canonical bundle;

- the Betti moduli space parametrising $G$-representations of the fundamental group of $C \backslash c$ with monodromy $e^{2 \pi i d / r}$ around $c$.

These moduli spaces are denoted respectively $M_{\mathrm{DR}}^{d}(C, G), M_{\mathrm{Dol}}^{d}(C, G)$ and $M_{\mathrm{B}}^{d}(C, G)$. For convenience, we simply write $M(C, G)$ when we refer indifferently to $M_{\mathrm{Dol}}^{0}(C, G)$, $M_{\mathrm{DR}}^{0}(C, G)$ or $M_{\mathrm{B}}^{0}(C, G)$.

In [9], Hausel and Thaddeus showed that the de Rham moduli spaces $M_{\mathrm{DR}}^{d}\left(C, \mathrm{SL}_{r}\right)$ and $M_{\mathrm{DR}}^{d}\left(C, \mathrm{PGL}_{r}\right)$ are mirror partners in the sense of Strominger-Yau-Zaslow mirror

\section{Communicated by Daniel Greb.}

\section{Mirko Mauri}

mauri@mpim-bonn.mpg.de

1 Max Planck Institute for Mathematics, Vivatgasse 7, 53111 Bonn, Germany 
symmetry. According to the general mirror symmetric framework, it is reasonable to expect a symmetry between their Hodge numbers.

Hausel and Thaddeus conjectured the equality of the stringy E-polynomials

$$
E_{\mathrm{st}}^{B^{e}}\left(M_{\mathrm{DR}}^{d}\left(C, \mathrm{SL}_{r}\right)\right)=E_{\mathrm{st}}^{\hat{B}^{d}}\left(M_{\mathrm{DR}}^{e}\left(C, \mathrm{PGL}_{r}\right)\right),
$$

for $(d, r)=(e, r)=1$, and they prove it for $r=2,3$. The conjecture is now a theorem due to [7] or [12].

In [8, Remark 3.30] Hausel asked what cohomology theory we should compute on $M_{\mathrm{DR}}^{d}\left(C, \mathrm{SL}_{r}\right)$, with $(d, r) \neq 1$, to accomplish the agreement (1).

We propose to use intersection cohomology. As first piece of evidence, we show the topological mirror symmetry conjecture in rank two, and degree zero, i.e. when we turn off the B-fields ${ }^{1} B$ and $\hat{B}$.

Theorem 0.1 (Topological mirror symmetry in rank two and degree zero) The intersection E-polynomial of $M\left(C, \mathrm{SL}_{2}\right)$ equals the stringy intersection E-polynomial of $M\left(C, \mathrm{PGL}_{2}\right)$

$$
\operatorname{IE}\left(M\left(C, \mathrm{SL}_{2}\right)\right)=I E_{\mathrm{st}}\left(M\left(C, \mathrm{PGL}_{2}\right)\right) .
$$

The refinements of the Hausel-Thaddeus conjecture postulated in [8, Conjecture 3.27] and [8, Conjecture 5.9] also hold true in rank two and degree zero, as long as we consider their intersection cohomology analogues; see Theorems 2.2 and 3.2.

\section{Intersection stringy E-polynomial}

The intersection cohomology of a complex variety $X$ with compact support, middle perversity and rational coefficients is denoted by $I H_{c}^{*}(X)$. Recall that $I H_{c}^{*}(X)$ carries a canonical mixed Hodge structure, and so we can define the intersection E-polynomial of $X$ as

$$
I E(X):=\sum_{r, s, d}(-1)^{d} \operatorname{dim}\left(\mathrm{Gr}_{r+s}^{W} I H_{c}^{d}(X, \mathbb{C})\right)^{r, s} u^{r} v^{s}
$$

Suppose that $X$ is endowed with the action of a finite abelian group $\Gamma$, and denote the group of characters of $\Gamma$ by $\hat{\Gamma}$. The intersection cohomology of $X$ decomposes under the action of $\Gamma$ into isotypic components:

$$
I H_{c}^{*}(X)=\bigoplus_{\kappa \in \hat{\Gamma}} I H^{*}(X)_{\kappa}
$$

Then, if we pose

$$
I E(X)_{\kappa}:=\sum_{r, s, d}(-1)^{d} \operatorname{dim}\left(\mathrm{Gr}_{r+s}^{W} I H_{c}^{d}(X, \mathbb{C})_{\kappa}\right)^{r, s} u^{r} v^{s},
$$

we obtain $I E(X)=\sum_{\kappa \in \hat{\Gamma}} I E(X)_{\kappa}$.

Define also the intersection stringy E-polynomial by

${ }^{1}$ See $[9, \S 4]$ for a definition of gerbe or B-field. 


$$
I E_{\mathrm{st}}(X):=\sum_{\gamma \in \Gamma} I E\left(X_{\gamma} / \Gamma ; u, v\right)(u v)^{F(\gamma)}
$$

where

- $X_{\gamma}$ is the fixed-point set of $\gamma \in \Gamma$.

- $F(\gamma)$ is the Fermionic shift, defined as $F(\gamma)=\sum_{j} w_{j}$, where $\gamma$ acts on the normal bundle of $X_{\gamma}$ in $X$ with eigenvalues $e^{2 \pi i w_{j}}$ with $w_{j} \in(0,1)$.

\section{Topological mirror symmetry}

Let $\Gamma:=\operatorname{Pic}^{0}(C)[r] \simeq(\mathbb{Z} / r \mathbb{Z})^{2 g}$ be the group of $r$-torsion line bundles over the compact Riemann surface $C$ of genus $g$, endowed with the canonical flat connection. The group $\Gamma$ acts by tensorisation on $M_{\mathrm{Dol}}^{d}\left(C, \mathrm{SL}_{r}\right)$ and $M_{\mathrm{DR}}^{d}\left(C, \mathrm{SL}_{r}\right)$. Via the non-abelian Hodge correspondence, the action corresponds to the algebraic action of the characters $\Gamma \simeq \operatorname{Hom}\left(\pi_{1}(C), \mathbb{Z} / r \mathbb{Z}\right)$ which acts on $M_{\mathrm{B}}^{d}\left(C, \mathrm{SL}_{r}\right)$ by multiplication. The quotient of $M^{d}\left(C, \mathrm{SL}_{r}\right)$ by the action of $\Gamma$ is isomorphic to $M^{d}\left(C, \mathrm{PGL}_{r}\right)$.

We identify $w: \Gamma \rightarrow \hat{\Gamma}$ through Poincaré duality (also known as Weil pairing)

$$
\Gamma \times \Gamma \simeq H_{1}(C, \mathbb{Z} / r \mathbb{Z}) \times H_{1}(C, \mathbb{Z} / r \mathbb{Z}) \rightarrow \mathbb{Z} / r \mathbb{Z} .
$$

Conjecture 2.1 (Topological mirror symmetry in degree zero) For $\kappa \in \hat{\Gamma}$ we have

$$
\operatorname{IE}\left(M\left(C, \mathrm{SL}_{r}\right)\right)_{\kappa}=\operatorname{IE}\left(M\left(C, \mathrm{SL}_{r}\right)_{\gamma} / \Gamma ; u, v\right)(u v)^{F(\gamma)}
$$

where $\gamma=w(\kappa)$. In particular, we obtain

$$
\operatorname{IE}\left(M\left(C, \mathrm{SL}_{r}\right)\right)=I E_{\mathrm{st}}\left(M\left(C, \mathrm{PGL}_{r}\right)\right) .
$$

Theorem 2.2 (Theorem 0.1) Conjecture 2.1 holds for $r=2$.

Proof Without loss of generality we can suppose $\gamma \neq 0$, or equivalently $\kappa \neq 1$. Indeed,

$$
\operatorname{IE}\left(M\left(C, \mathrm{SL}_{r}\right)\right)_{1}=\operatorname{IE}\left(M\left(C, \mathrm{SL}_{r}\right) / \Gamma ; u, v\right) ;
$$

see for instance the proof of [6, Proposition 3].

For any $\gamma \in \Gamma \backslash\{0\}$, we have an associated 2-torsion line bundle $L_{\gamma}$. Consider the étale double cover $\pi_{\gamma}: C_{\gamma} \rightarrow C$ consisting of the square root of a non-zero section of $L_{\gamma}^{\otimes 2} \simeq \mathcal{O}_{C}$ in the total space of $L_{\gamma}$, and let $\imath$ be its deck transformation.

For any $L \in M\left(C_{\gamma}, \mathrm{GL}_{1}\right)$, the rank-two vector bundle $L \oplus \imath^{*} L$ is a $l$-invariant object in $M\left(C_{\gamma}, \mathrm{GL}_{2}\right)$, which descends to an object $L_{l} \in M\left(C, \mathrm{GL}_{2}\right)$. Hence, the pushforward morphism

$$
\pi_{\gamma, *}: M\left(C_{\gamma}, \mathrm{GL}_{1}\right) \rightarrow M\left(C, \mathrm{GL}_{2}\right), \quad L \mapsto L_{l},
$$

descends to a $\Gamma$-invariant embedding

$$
j: M\left(C_{\gamma}, \mathrm{GL}_{1}\right) / \mathbb{Z} / 2 \mathbb{Z} \hookrightarrow M\left(C, \mathrm{GL}_{2}\right) .
$$

The determinant map det $\pi_{\gamma, *}$ can be identified with the norm map 


$$
\mathrm{Nm}_{C_{\gamma} / C}: M\left(C_{\gamma}, \mathrm{GL}_{1}\right) / \mathbb{Z} / 2 \mathbb{Z} \rightarrow M\left(C, \mathrm{GL}_{1}\right), \quad L \mapsto L \otimes \imath^{*} L,
$$

Therefore, the fixed-point set $M\left(C, \mathrm{SL}_{2}\right)_{\gamma}$ admits the following geometric characterization:

$$
M\left(C, \mathrm{SL}_{2}\right)_{\gamma}=\operatorname{Im} j \cap M\left(C, \mathrm{SL}_{2}\right) \simeq \operatorname{ker} \mathrm{Nm}_{C_{\gamma} / C}^{\circ},
$$

where the last term is the connected component of $\mathrm{Nm}_{C_{\gamma} / C}^{-1}\left(\mathcal{O}_{C}\right)$ containing $\mathcal{O}_{C_{\gamma}}$.

On the Dolbeault side, $M_{\text {Dol }}\left(C, \mathrm{SL}_{2}\right)_{\gamma}$ is isomorphic to the quotient by $\mathbb{Z} / 2 \mathbb{Z}$ of the cotangent bundle of an abelian variety of dimension $g-1$, as $M_{\mathrm{Dol}}\left(C_{\gamma}, \mathrm{GL}_{1}\right)$ is isomorphic to $T^{*} \mathrm{Pic}^{0}\left(C_{\gamma}\right)$; see also the proof of Theorem 3.2. On the Betti side, we have

$$
M_{\mathrm{B}}\left(C_{\gamma}, \mathrm{GL}_{1}\right) \simeq\left(\mathbb{C}^{*}\right)^{4 g-2} \text {, and so } M_{\mathrm{B}}\left(C, \mathrm{SL}_{2}\right)_{\gamma} \simeq\left(\mathbb{C}^{*}\right)^{2 g-2} / \mathbb{Z} / 2 \mathbb{Z} \text {. }
$$

The involution defining the $\mathbb{Z} / 2 \mathbb{Z}$-quotient is the inverse of the group law.

Since the $\Gamma$-module $I H_{c}^{*}\left(M\left(C, \mathrm{SL}_{2}\right)\right)$ is a direct sums of copies of the trivial and regular representations by [13, Remark 4.4], we have

$$
\operatorname{IE}\left(M\left(C, \mathrm{SL}_{2}\right)\right)_{\kappa}=\operatorname{IE}\left(M\left(C, \mathrm{SL}_{2}\right)\right)_{\kappa^{\prime}}
$$

for any $\kappa, \kappa^{\prime} \in \hat{\Gamma} \backslash\{1\}$. Thanks to [13, Corollary 1.11, Equations (23) and (25)] we have

$$
\begin{aligned}
I E\left(M_{\mathrm{Dol}}\left(C, \mathrm{SL}_{2}\right)\right)_{\kappa} & =\frac{1}{2}(u v)^{3 g-3}\left((u+1)^{g-1}(v+1)^{g-1}+(u-1)^{g-1}(v-1)^{g-1}\right) \\
& =\operatorname{IE}\left(M_{\mathrm{Dol}}\left(C, \mathrm{SL}_{2}\right)_{\gamma} / \Gamma\right)(u v)^{2 g-2}, \\
\operatorname{IE}\left(M_{\mathrm{B}}\left(C, \mathrm{SL}_{2}\right)\right)_{\kappa} & =\frac{1}{2}(u v)^{2 g-2}\left((u v+1)^{2 g-2}+(u v-1)^{2 g-2}\right) \\
& =\operatorname{IE}\left(\left(\mathbb{C}^{*}\right)^{2 g-2} / \mathbb{Z} / 2 \mathbb{Z}\right)(u v)^{2 g-2} \\
& =I E\left(M_{\mathrm{B}}\left(C, \mathrm{SL}_{2}\right)_{\gamma} / \Gamma\right)(u v)^{2 g-2} .
\end{aligned}
$$

Note that the Fermionic shift $F(\gamma)$ equals half of the codimension of $M_{\mathrm{Dol}}\left(C, \mathrm{SL}_{2}\right)_{\gamma}$ in $M_{\text {Dol }}\left(C, \mathrm{SL}_{2}\right)$, since $\gamma$ is an involution. Hence, for $\gamma \neq 0$ we have indeed

$$
F(\gamma)=\frac{1}{2} \operatorname{codim} M_{\text {Dol }}\left(C, \mathrm{SL}_{2}\right)_{\gamma}=2 g-2 .
$$

Finally, the same argument of $[9, \S 6]$, together with [5, Theorem 3.2], implies that Conjecture (3) for the Dolbeault moduli spaces yields (3) for the de Rham moduli spaces.

Remark 2.3 (Failure of topological mirror symmetry for ordinary cohomology) In general the equality (3) fails for ordinary cohomology. For instance, for $\kappa \neq 1, \gamma=w(\kappa)$ and $q=u v$ we have

$$
\begin{aligned}
E\left(M_{B}\left(C, \mathrm{SL}_{2}\right)\right)_{K} & =\frac{1}{2} q^{2 g-2}\left((q+1)^{2 g-2}+(q-1)^{2 g-2}-2\right) \\
& \neq \frac{1}{2} q^{2 g-2}\left((q+1)^{2 g-2}+(q-1)^{2 g-2}\right)=E\left(M_{B}\left(C, \mathrm{SL}_{2}\right)_{\gamma} / \Gamma\right) q^{F(\gamma)},
\end{aligned}
$$

where the first equality follows from [10, Theorem 2] or [1, Theorem 1.3], together with [13, Remark 4.3], while the last equality comes from (4), since $M_{B}\left(C, \mathrm{SL}_{2}\right)_{\gamma} / \Gamma$ has only quotient singularities. This shows that there is a non-negligible contribution of the singularity of $M\left(C, \mathrm{SL}_{r}\right)$ to the agreement (3) of Hodge numbers. 
Remark 2.4 The proof of Theorem 2.2 relies on the computation of $\sum_{\kappa \neq 1} \operatorname{IE}\left(M\left(C, \mathrm{SL}_{2}\right)\right)_{\kappa}$ in [13, Corollary 1.11], and ultimately on the explicit construction of a desingularization of $M\left(C, \mathrm{SL}_{2}\right)$ in $[13, \S 3]$. To the best of the author's knowledge, this is not available in higher rank, and so it is unclear if the arguments above extend in higher rank. However, remarkable progress in this direction have been made in [12] and [11].

\section{Perverse topological mirror symmetry}

The intersection cohomology of $M_{\mathrm{Dol}}\left(C, \mathrm{SL}_{r}\right)$ and $M_{\mathrm{Dol}}\left(C, \mathrm{SL}_{2}\right)_{\gamma}$ are filtered by the perverse filtration $P$ associated to the Hitchin fibrations

$$
\begin{gathered}
\chi: M_{\mathrm{Dol}}\left(C, \mathrm{SL}_{r}\right) \rightarrow \Lambda:=\bigoplus_{i=2}^{n} H^{0}\left(C, K_{C}^{\otimes i}\right) \\
\chi_{\gamma}:=\left.\chi\right|_{M_{\mathrm{Dol}}\left(C, \mathrm{SL}_{r}\right)_{\gamma}}: M_{\mathrm{Dol}}\left(C, \mathrm{SL}_{r}\right)_{\gamma} \rightarrow \Lambda_{\gamma}:=\operatorname{Im}\left(\left.\chi\right|_{\left.M_{\mathrm{Dol}}\left(C, \mathrm{SL}_{r}\right)_{\gamma}\right) \subseteq \Lambda,}\right.
\end{gathered}
$$

which assigns to the Higgs bundle $(E, \phi)$ the characteristic polynomial of $\phi$; see $[13, \S 2.2]$ for a brief account on the perverse filtration.

Recall that the graded pieces of the perverse filtration carries a canonical mixed Hodge structure; see for instance $[4, \S 7]$. Thus we can define the perverse intersection E-polynomial

$$
\begin{aligned}
\operatorname{PIE}\left(M_{\mathrm{Dol}}\left(C, \mathrm{SL}_{r}\right)\right. & ; u, v, q) \\
& :=\sum_{r, s, d}(-1)^{d} \operatorname{dim}\left(\mathrm{Gr}_{r+s}^{W} \mathrm{Gr}_{k}^{P} I H_{c}^{d}\left(M_{\mathrm{Dol}}\left(C, \mathrm{SL}_{r}\right), \mathbb{C}\right)\right)^{r, s} u^{r} v^{s} q^{k}
\end{aligned}
$$

and the stringy perverse intersection E-polynomial

$$
P I E_{\mathrm{st}}\left(M_{\mathrm{Dol}}\left(C, \mathrm{PGL}_{r}\right) ; u, v, q\right):=\sum_{\gamma \in \Gamma} \operatorname{PIE}\left(M_{\mathrm{Dol}}\left(C, \mathrm{SL}_{r}\right)_{\gamma} / \Gamma ; u, v\right)(u v q)^{F(\gamma)} .
$$

By Definition (2) and the last paragraph of the proof of Theorem 2.2, we have

$$
\operatorname{PIE}\left(M_{\mathrm{Dol}}\left(C, \mathrm{SL}_{r}\right) ; u, v, 1\right)=\operatorname{IE}\left(M_{\mathrm{Dol}}\left(C, \mathrm{SL}_{r}\right) ; u, v\right)=I E\left(M_{\mathrm{DR}}\left(C, \mathrm{SL}_{r}\right) ; u, v\right) .
$$

Further, Relative Hard Lefschetz [3, Theorem 2.1.1] implies

$$
P I E\left(M_{\mathrm{Dol}}\left(C, \mathrm{SL}_{r}\right) ; u, v, q\right)=(u v q)^{\operatorname{dim}} \operatorname{PIE}\left(M_{\mathrm{Dol}}\left(C, \mathrm{SL}_{r}\right) ; u, v, \frac{1}{u v q}\right),
$$

where $\operatorname{dim}=2\left(r^{2}-1\right)(g-1)$.

We conjecture the exchange of the perverse Hodge numbers.

Conjecture 3.1 (Perverse topological mirror symmetry in degree zero)

$$
\operatorname{PIE}\left(M_{\mathrm{Dol}}\left(C, \mathrm{SL}_{r}\right) ; u, v, q\right)=(u v q)^{\operatorname{dim}} P I E_{\mathrm{str}}\left(M_{\mathrm{Dol}}\left(C, \mathrm{PGL}_{r}\right) ; u, v, \frac{1}{u v q}\right) .
$$

For $q=1$, Conjecture 3.1 specialises to

$$
I E\left(M_{\mathrm{Dol}}\left(C, \mathrm{SL}_{r}\right)\right)=I E_{\mathrm{st}}\left(M_{\mathrm{Dol}}\left(C, \mathrm{PGL}_{r}\right)\right) .
$$


Further, the PI=WI conjecture [2, Question 4.1.7] would imply

$$
\operatorname{PIE}\left(M_{\mathrm{Dol}}\left(C, \mathrm{SL}_{r}\right) ; 1,1, q\right)=\operatorname{IE}\left(M_{\mathrm{B}}\left(C, \mathrm{SL}_{r}\right) ; q\right),
$$

and together with Conjecture 3.1 it would give

$$
I E\left(M_{\mathrm{B}}\left(C, \mathrm{SL}_{r}\right)\right)=I E_{\mathrm{st}}\left(M_{\mathrm{B}}\left(C, \mathrm{PGL}_{r}\right)\right) .
$$

Theorem 3.2 Conjecture 3.1 holds for $r=2$.

Proof By Relative Hard Lefschetz, it is enough to show

$$
\operatorname{PIE}\left(M_{\mathrm{Dol}}\left(C, \mathrm{SL}_{2}\right)\right)_{\kappa}=\operatorname{PIE}\left(M_{\mathrm{Dol}}\left(C, \mathrm{SL}_{2}\right)_{\gamma} / \Gamma ; u, v\right)(u v q)^{F(\gamma)}
$$

for any $\kappa \in \hat{\Gamma}$ and $\gamma=w(\kappa)$. As in Theorem 2.2, the case $\kappa=1$, alias $\gamma=0$, is trivial. Suppose then $\kappa \neq 1$ and $\gamma \neq 0$. The perverse filtration on $I H^{d}\left(M_{\mathrm{Dol}}\left(C, \mathrm{SL}_{2}\right)\right)_{K}$ is concentrated in degree $d-2 g+2$ by [13, Theorem 5.5]. Moreover, the Hitchin map $\chi_{\gamma}$ is a quotient by the inverse of the group law of the projection

$$
M_{\mathrm{Dol}}\left(C_{\gamma}, \mathrm{GL}_{1}\right) \supset T^{*} \operatorname{Prym}=\operatorname{Prym} \times \mathbb{C}^{g-1} \rightarrow \mathbb{C}^{g-1},
$$

where Prym is the connected component of the identity of the kernel of the norm map $\mathrm{Nm}: \operatorname{Pic}^{0}\left(C_{\gamma}\right) \rightarrow \operatorname{Pic}^{0}(C)$, given by $\mathrm{Nm}(L)=L \otimes \imath^{*} L$. Hence, the perverse filtration on $I H^{d}\left(M_{\mathrm{Dol}}\left(C, \mathrm{SL}_{2}\right)_{\gamma} / \Gamma\right)$, with $\gamma \neq 0$, is concentrated in degree $d$; cf proof of [5, Theorem 6.6]. Then one easily see that Conjecture 3.1 for $r=2$ is equivalent to Theorem 2.2.

Acknowledgements This work have been supported by the Max Planck Institute for Mathematics. We thank an anonymous referee for helpful comments.

Funding Open Access funding enabled and organized by Projekt DEAL.

Open Access This article is licensed under a Creative Commons Attribution 4.0 International License, which permits use, sharing, adaptation, distribution and reproduction in any medium or format, as long as you give appropriate credit to the original author(s) and the source, provide a link to the Creative Commons licence, and indicate if changes were made. The images or other third party material in this article are included in the article's Creative Commons licence, unless indicated otherwise in a credit line to the material. If material is not included in the article's Creative Commons licence and your intended use is not permitted by statutory regulation or exceeds the permitted use, you will need to obtain permission directly from the copyright holder. To view a copy of this licence, visit http://creativecommons.org/licenses/by/4.0/.

\section{References}

1. Baraglia, D., Hekmati, P.: Arithmetic of singular character varieties and their E-polynomials. Proc. Lond. Math. Soc. (3) 114(2), 293-332 (2017)

2. de Cataldo, M.A., Maulik, D.: The perverse filtration for the Hitchin fibration is locally constant. Pure Appl. Math. Q. 16(5),1441-1464 (2020)

3. de Cataldo, M.A., Migliorini, L.: The Hodge theory of algebraic maps. Ann. Sci. École Norm. Sup. (4) 38(5), 693-750 (2005)

4. de Cataldo, M.A., Migliorini, L.: The perverse filtration and the Lefschetz hyperplane theorem. Ann. Math. (2) 171(3), 2089-2113 (2010)

5. Felisetti, C., Mauri, M.: $\mathrm{P}=\mathrm{W}$ conjectures for character varieties with symplectic resolution. arXiv:2006. 08752 (2020) 
6. Göttsche, L., Soergel, W.: Perverse sheaves and the cohomology of Hilbert schemes of smooth algebraic surfaces. Math. Ann. 296(2), 235-245 (1993)

7. Groechenig, M., Wyss, D., Ziegler, P.: Mirror symmetry for moduli spaces of Higgs bundles via p-adic integration. Invent. Math. 221(2), 505-596 (2020)

8. Hausel, T.: Global topology of the Hitchin system. In: Handbook of moduli. Vol. II, volume 25 of Adv. Lect. Math. (ALM), pp. 29-69. Int. Press, Somerville (2013)

9. Hausel, T., Thaddeus, M.: Mirror symmetry, Langlands duality, and the Hitchin system. Invent. Math. 153(1), 197-229 (2003)

10. Martínez, J., Muñoz, V.: E-polynomials of the SL(2, C)-character varieties of surface groups. Int. Math. Res. Not. IMRN 3, 926-961 (2016)

11. Maulik, D., Shen, J.: Cohomological $\chi$-independence for moduli of one-dimensional sheaves and moduli of Higgs bundles. arXiv:2012.06627 (2020)

12. Maulik, D., Shen, J.: Endoscopic decompositions and the Hausel-Thaddeus conjecture. to appear at Forum Math. Pi. arXiv:2008.08520 (2020)

13. Mauri, M.: Intersection cohomology of rank two character varieties of surface groups. arXiv:2101.04628 (2021)

14. Simpson, C.T.: Moduli of representations of the fundamental group of a smooth projective variety. II. Inst. Hautes Études Sci. Publ. Math. 80, 5-79 (1995)

Publisher's Note Springer Nature remains neutral with regard to jurisdictional claims in published maps and institutional affiliations. 